\title{
Fractional Differential Equations with Initial Conditions at Inner Points in Banach Spaces
}

\author{
Xiaoping $\mathrm{Xu}^{1}$, Guangxian $\mathrm{Wu}^{2}$, Qixiang Dong3 \\ ${ }^{1}$ Department of Basic Course, Nantong Vocational University, Nantong, China \\ ${ }^{2}$ Department of Mathematics, Jiaozuo Teacher's College, Jiaozuo, China \\ ${ }^{3}$ School of Mathematical Sciences, Yangzhou University, Yangzhou, China \\ Email:xxp@mail.ntvu.edu.cn, guangxian08@126.com, qxdongyz@outlook.com, qxdong@yzu.edu.cn
}

Received 12 June 2015; accepted 4 December 2015; published 7 December 2015

Copyright (@ 2015 by authors and Scientific Research Publishing Inc.

This work is licensed under the Creative Commons Attribution International License (CC BY).

http://creativecommons.org/licenses/by/4.0/

(c) (i) Open Access

\section{Abstract}

This paper is concerned with nonlinear fractional differential equations with the Caputo fractional derivatives in Banach spaces. Local existence results are obtained for initial value problems with initial conditions at inner points for the cases that the nonlinear parts are Lipschitz and non-Lipschitz, respectively. Hausdorff measure of non-compactness and Darbo-Sadovskii fixed point theorem are employed to deal with the non-Lipschitz case. The results obtained in this paper extend the classical Peano's existence theorem for first order differential equations partly to fractional cases.

Keywords

Fractional Derivative, Differential Equation, Initial Value Problem, Measure of Non-Compactness

\section{Introduction}

Let $\mathbf{X}$ be a Banach space. We consider the nonlinear fractional differential equation

$$
{ }^{c} D_{a}^{\alpha} y(x)=f(x, y(x)), \quad x \geq x_{0} \in(a, b),
$$

with the initial value condition at an inner point (IVP for short)

$$
y\left(x_{0}\right)=y_{0},
$$

where $0<\alpha \leq 1,{ }^{c} D_{a}^{\alpha}$ is the Caputo fractional derivative, $f:[a, b] \times \mathbf{X} \rightarrow \mathbf{X}$ is a given function satisfying some assumptions that will be specified later.

Fractional differential equations arise in many engineering and scientific disciplines as the mathematical 
modeling of systems and processes in the fields of physics, chemistry, biology, economics, control theory, signal and image processing, etc. which involve fractional order derivatives. Fractional differential equations also serve as an excellent tool for the description of hereditary properties of various materials and processes. Consequently, the subject of fractional differential equations is gaining much importance and attention (see [1]-[5]). There are a large number of papers dealing with the existence or properties of solutions to fractional differential equations. For an extensive collection of such results, we refer the reader to the monograph [1] and [3] and references therein.

In the most of the mentioned works above, the initial value problems for fractional differential equations were studied with the initial conditions at the endpoints of the definition interval, recalling that the classical existence and uniqueness theorem are for first order differential equations, where the initial conditions are at any inner points of the considered interval. On the other hand, classical integer order derivatives at a point are determined by some neighbourhoods of this point, while the fractional derivatives are determined by intervals from the endpoints up to this point. Fractional derivatives at the same point with different endpoints of the definition intervals are in fact different derivatives. Let us investigate the fractional differential equations

$$
{ }^{c} D_{0}^{\alpha} y_{1}(x)=x^{2}, \quad x \geq 1
$$

and

$$
{ }^{c} D_{1}^{\alpha} y_{2}(x)=x^{2}, \quad x \geq 1
$$

with $0<\alpha<1$ and the same initial value condition

$$
y_{1}(1)=y_{2}(1)=\frac{2}{\Gamma(3+\alpha)} .
$$

A direct computation deduces that the solutions to the above initial value problems are

$$
y_{1}(x)=\frac{2 x^{2+\alpha}}{\Gamma(3+\alpha)}
$$

and

$$
y_{2}(x)=\frac{2(x-1)^{2+\alpha}}{\Gamma(3+\alpha)}+\frac{2(x-1)^{1+\alpha}}{\Gamma(2+\alpha)}+\frac{(x-1)^{\alpha}}{\Gamma(1+\alpha)}+\frac{2}{\Gamma(3+\alpha)}
$$

respectively. By a numerical method, we can find that $y_{1}(x) \neq y_{2}(x)$ for $x>1$. This example shows that ${ }^{c} D_{0}^{\alpha}$ and ${ }^{c} D_{1}^{\alpha}$ are two different "fractional derivatives", and Equations (1.3) and (1.4) are two different equations.

Motivated by the above comment, in this paper, we study the existence of solutions to the nonlinear Caputo fractional differential equation modeled as (1.1), with the initial conditions at inner points of the definition interval of the fractional derivative. In this case, the equivalent integral equation is a Volterra-Fredholm equation. Local existence results are obtained for the cases that the function $f$ on the righthand side of the equation is Lipschitz and Caratheodory type, respectively. The theory of measure of non-compactness is employed to deal with the non-Lipschitz case. In this sense, the classical Peano's theorem is extended to fractional cases.

\section{Preliminaries and Lemmas}

In this section we collect some definitions and results needed in our further investigations.

Let $C([a, b] ; \mathbf{X})$ be the Banach space of all continuous functions $u:[a, b] \rightarrow \mathbf{X}$ with the norm $\|u\|_{\infty}=\sup \{\|u(x)\|: x \in[a, b]\}$, and $L^{p}((a, b) ; \mathbf{X})$ the Banach space of all measurable functions $u:[a, b] \rightarrow \mathbf{X}$ such that $\|u(\cdot)\|^{p}:[a, b] \rightarrow \mathbf{R}^{+}$are Lebesgue integrable, equipped with the norm $\|u\|_{p}=\left(\int_{a}^{b}\|u(x)\|^{p} \mathrm{~d} x\right)^{1 / p}$ with $p \geq 1$.

Definition 2.1 ([1]): Let $\alpha>0$ be a fixed number. The Riemann-Liouville fractional integral of order $\alpha>0$ of the function $h:[a, b] \rightarrow \mathbf{R}$ is defined by 


$$
I_{a}^{\alpha} h(x)=\frac{1}{\Gamma(\alpha)} \int_{a}^{x}(x-t)^{\alpha-1} h(t) \mathrm{d} t, x \in[a, b]
$$

where $\Gamma(\cdot)$ denotes the Gamma function, i.e., $\Gamma(z)=\int_{0}^{\infty} \mathrm{e}^{-t} t^{z-1} \mathrm{~d} t$.

It has been shown that the fractional integral operator $I_{a}^{\alpha}$ transforms the space $L^{1}((a, b) ; \mathbf{R})$ into $C([a, b] ; \mathbf{R})$, and some other properties of $I_{a}^{\alpha}$ are refered to [1].

Definition 2.2 ([1]): Let $h:[a, b] \rightarrow \mathbf{R}, \alpha>0$, and $m=[\alpha]+1$. The Caputo fractional derivative of order $\alpha$ of $h$ at the point $x$ is defined by

$$
{ }^{c} D_{a}^{\alpha} h(x)=\frac{1}{\Gamma(m-\alpha)} \int_{a}^{x}(x-t)^{m-\alpha-1} h^{(m)}(t) \mathrm{d} t, \quad x \in[a, b] .
$$

${ }^{c} D_{a}^{\alpha}$ is also called the Caputo fractional differential operator.

Lemma 2.1 ([1]): Let $0<\alpha<1$ and $f \in C^{1}[a, b]$. Then

$$
I_{a}^{\alpha} D_{a}^{\alpha} f(t)=f(t)-f(a)
$$

for $t \in[a, b]$.

In recent decades measures of noncompactness play very important role in nonlinear analysis [6]-[9]. They are often applied to the theories of differential and integral equations as well as to the operator theory and geometry of Banach spaces ([10]-[15]). One of the most important examples of measure of noncompactness is the Hausdorff's measure of noncompactness $\beta_{Y}$, which is defined by

$$
\beta_{Y}(B)=\inf \{r>0 ; B \text { can be covered with a finite number of balls of radius equal to } r\}
$$

for bounded set $B$ in a Banach space $Y$.

The following properties of Hausdorff's measure of noncompactness are well known.

Lemma 2.2 ([8]): Let $Y$ be a real Banach space and $B, C \subseteq Y$ be bounded,the following properties are satisfied:

(1) $B$ is pre-compact if and only if $\beta_{Y}(B)=0$;

(2) $\beta_{Y}(B)=\beta_{Y}(\bar{B})=\beta_{Y}(\operatorname{conv} B)$ where $\bar{B}$ and $\operatorname{conv} B$ mean the closure and convex hull of $B$ respectively;

(3) $\beta_{Y}(B) \leq \beta_{Y}(C)$ when $B \subseteq C$;

(4) $\beta_{Y}(B+C) \leq \beta_{Y}(B)+\beta_{Y}(C)$ where $B+C=\{x+y ; x \in B, y \in C\}$;

(5) $\beta_{Y}(B \cup C) \leq \max \left\{\beta_{Y}(B), \beta_{Y}(C)\right\}$;

(6) $\beta_{Y}(\lambda B)=|\lambda| \beta_{Y}(B)$ for any $\lambda \in R$;

(7) If the map $Q: D(Q) \subseteq Y \rightarrow Z$ is Lipschitz continuous with constant $k$ then $\beta_{Z}(Q B) \leq k \beta_{Y}(B)$ for any bounded subset $B \subseteq D(Q)$, where $Z$ be a Banach space;

(8) $\beta_{Y}(B)=\inf \left\{d_{Y}(B, C) ; C \subseteq Y\right.$ be precompact $\}=\inf \left\{d_{Y}(B, C) ; C \subseteq Y\right.$ be finite valued $\}$, where

$d_{Y}(B, C)$ means the nonsymmetric (or symmetric) Hausdorff distance between $B$ and $C$ in $Y$;

(9) If $\left\{W_{n}\right\}_{n=1}^{+\infty}$ is a decreasing sequence of bounded closed nonempty subsets of $Y$ and $\lim _{n \rightarrow+\infty} \beta_{Y}\left(W_{n}\right)=0$, then $\bigcap_{n=1}^{+\infty} W_{n}$ is nonempty and compact in $Y$.

The map $Q: W \subseteq Y \rightarrow Y$ is said to be a $\beta_{Y}$-contraction if there exists a positive constant $k<1$ such that $\beta_{Y}(Q(B)) \leq k \beta_{Y}(B)$ for any bounded closed subset $B \subseteq W$, where $Y$ is a Banach space.

Lemma 2.3 ([8]): (Darbo-Sadovskii) If $W \subseteq Y$ is bounded closed and convex, the continuous map $Q: W \rightarrow W$ is a $\beta_{Y}$-contraction, then the map $Q$ has at least one fixed point in $W$.

In this paper we denote by $\beta$ the Hausdorff's measure of noncompactness of $X$ and by $\beta_{c}$ the Hausdorff's measure of noncompactness of $C([a, b] ; X)$. To discuss the existence we need the following lemmas in this paper.

Lemma 2.4 ([8]): If $W \subseteq C([a, b] ; X)$ is bounded, then

$$
\beta(W(t)) \leq \beta_{c}(W)
$$


for all $t \in[a, b]$, where $W(t)=\{u(t) ; u \in W\} \subseteq X$. Furthermore if $W$ is equicontinuous on [a,b], then $\beta(W(t))$ is continuous on $[a, b]$ and

$$
\beta_{c}(W)=\sup \{\beta(W(t)), t \in[a, b]\} .
$$

Lemma 2.5 ([14] [15]): If $\left\{u_{n}\right\}_{n=1}^{\infty} \subset L^{1}(a, b ; X)$ is uniformly integrable, then $\beta\left(\left\{u_{n}(t)\right\}_{n=1}^{\infty}\right)$ is measurable and

$$
\beta\left(\left\{\int_{a}^{t} u_{n}(s) \mathrm{d} s\right\}_{n=1}^{\infty}\right) \leq 2 \int_{a}^{t} \beta\left(\left\{u_{n}(s)\right\}_{n=1}^{\infty}\right) \mathrm{d} s .
$$

Lemma 2.6 ([8]): If $W \subseteq C([a, b] ; X)$ is bounded and equicontinuous, then $\beta(W(s))$ is continuous and

$$
\beta\left(\int_{a}^{t} W(s) \mathrm{d} s\right) \leq \int_{a}^{t} \beta(W(s)) \mathrm{d} s
$$

for all $t \in[a, b]$, where $\int_{a}^{t} W(s) \mathrm{d} s=\left\{\int_{a}^{t} x(s) \mathrm{d} s: x \in W\right\}$.

\section{Existence Results}

In this section, we study the initial value problem for nonlinear fractional differential equations with initial conditions at inner points. More precisely, we will prove a Peano type theorem of the fractional version. We begin with the definition of the solutions to this problem. Consider initial value problem

$$
\left\{\begin{array}{l}
{ }^{c} D_{a}^{\alpha} y(x)=h(x), \quad x \geq x_{0} \in(a, b), \\
y\left(x_{0}\right)=y_{0} .
\end{array}\right.
$$

Since the fractional derivative of a function $y$ at an inner point $x \in(a, b)$ is determined by the values of $y$ on the interval $[a, x]$, for $h>0$ and $x \in\left[x_{0}, x_{0}+h\right]$, we get from Lemma 2.3 that

$$
y(x)-y(a)=\frac{1}{\Gamma(\alpha)} \int_{a}^{x}(x-t)^{\alpha-1} h(t) \mathrm{d} t .
$$

The initial condition then implies that

$$
y(a)=y_{0}-\frac{1}{\Gamma(\alpha)} \int_{a}^{x_{0}}\left(x_{0}-t\right)^{\alpha-1} h(t) \mathrm{d} t .
$$

Inserting this into (3.2) we obtain

$$
y(x)=y_{0}+\frac{1}{\Gamma(\alpha)}\left(\int_{a}^{x}(x-t)^{\alpha-1} h(t) \mathrm{d} t-\int_{a}^{x_{0}}\left(x_{0}-t\right)^{\alpha-1} h(t) \mathrm{d} t\right) .
$$

Based on the above analysis (see [1]), we give the definition of mild solutions to the IVP (1.1)-(1.2).

Definition 3.1: A contionuous function $y \in C\left(\left[a, x_{0}+h\right] ; \mathbf{X}\right)$ is said to be a mild solution to (1.1)-(1.2) if it satisfies

$$
y(x)=y_{0}+\frac{1}{\Gamma(\alpha)}\left(\int_{a}^{x}(x-t)^{\alpha-1} f(t, y(t)) \mathrm{d} t-\int_{a}^{x_{0}}\left(x_{0}-t\right)^{\alpha-1} f(t, y(t)) \mathrm{d} t\right),
$$

where $h>0$ and $x_{0}+h \in[a, b]$.

We first give an existence result based on the Banach contraction principle.

Theorem 3.1: Let $0<\alpha<1$, and $G=[a, b] \times \mathbf{X}$. Let $f: G \rightarrow \mathbf{X}$ be continuous and fulfil a Lipschitz condition with respect to the second variable with a Lipschitz constant $L$, i.e.

$$
\left|f\left(x, y_{2}\right)-f\left(x, y_{1}\right)\right| \leq L\left\|y_{2}-y_{1}\right\|, \quad\left(x, y_{1}\right),\left(x, y_{2}\right) \in G .
$$

Then for $\left(x_{0}, y_{0}\right) \in G$ with $x_{0}<a+\left(\frac{\Gamma(\alpha+1)}{2 L}\right)^{1 / \alpha}$, there exist an $h>0$ with $x_{0}+h \in[a, b]$ and a unique 
mild solution $y \in C\left(\left[a, x_{0}+h\right] ; \mathbf{X}\right)$ to the IVP (1.1)-(1.2).

Proof. Since $x_{0}<a+\left(\frac{\Gamma(\alpha+1)}{2 L}\right)^{1 / \alpha}$, we can take an $h>0$ with $x_{0}+h \in[a, b]$ such that

$$
x_{0}+h<a+\left(\frac{\Gamma(\alpha+1)}{2 L}\right)^{1 / \alpha} .
$$

We define a mapping $T: C\left(\left[a, x_{0}+h\right], \mathbb{X}\right) \rightarrow C\left(\left[a, x_{0}+h\right], \mathbb{X}\right)$ by

$$
(T y)(x)=y_{0}+\int_{a}^{x} \frac{(x-t)^{\alpha-1}}{\Gamma(\alpha)} f(t, y(t)) \mathrm{d} t-\int_{a}^{x_{0}} \frac{\left(x_{0}-t\right)^{\alpha-1}}{\Gamma(\alpha)} f(t, y(t)) \mathrm{d} t
$$

for $y \in C\left(\left[a, x_{0}+h\right], \mathbb{X}\right)$ and $x \in\left[a, x_{0}+h\right]$. Then for any $y_{1}, y_{2} \in C\left(\left[a, x_{0}+h\right], \mathbb{X}\right)$ and $x \in\left[a, x_{0}+h\right]$, we have

$$
\begin{aligned}
& \left\|\left(T y_{2}\right)(x)-\left(T y_{1}\right)(x)\right\| \\
& \leq \int_{a}^{x} \frac{(x-t)^{\alpha-1}}{\Gamma(\alpha)}\left\|f\left(t, y_{2}(t)\right)-f\left(t, y_{1}(t)\right)\right\| \mathrm{d} t \\
& \quad+\int_{a}^{x_{0}} \frac{\left(x_{0}-t\right)^{\alpha-1}}{\Gamma(\alpha)}\left\|f\left(t, y_{2}(t)\right)-f\left(t, y_{1}(t)\right)\right\| \mathrm{d} t \\
& \leq L \int_{a}^{x} \frac{(x-t)^{\alpha-1}}{\Gamma(\alpha)}\left\|y_{2}(t)-y_{1}(t)\right\| \mathrm{d} t+L \int_{a}^{x_{0}} \frac{\left(x_{0}-t\right)^{\alpha-1}}{\Gamma(\alpha)}\left\|y_{2}(t)-y_{1}(t)\right\| \mathrm{d} t \\
& \leq \frac{2 L\left(x_{0}-a\right)^{\alpha}}{\Gamma(\alpha+1)}\left\|y_{2}-y_{1}\right\|_{\infty} .
\end{aligned}
$$

It then follows that

$$
\left\|T y_{2}-T y_{1}\right\|_{\infty} \leq K\left\|y_{2}-y_{1}\right\|_{\infty}
$$

with $K=\frac{2 L\left(x_{0}+h-a\right)^{\alpha}}{\Gamma(\alpha+1)}$. Since $x_{0}+h<a+\left(\frac{\Gamma(\alpha+1)}{2 L}\right)^{1 / \alpha}$, we get that $\frac{2 L\left(x_{0}+h-a\right)^{\alpha}}{\Gamma(\alpha+1)}<1$. Thus an application of Banach's fixed point theorem yields the existence and uniqueness of solution to our integral equation (3.3).

Remark 3.1: The condition $x_{0}<a+\left(\frac{\Gamma(\alpha+1)}{2 L}\right)^{1 / \alpha}$ means that the point $x_{0}$ cannot be far away from $a$. However, the following example shows that we cannot expect that there exists a solution to (1.1)-(1.2) for each $x_{0} \in(a, b)$.

Example 3.1: Considering the differential equation with the Caputo fractional derivative

$$
{ }^{c} D_{0}^{1 / \alpha} y(x)=\frac{2 \sqrt{x}}{\sqrt{\pi c}} y^{2}(x),
$$

where $c>0$ is a constant. A direct computation shows that it admits a solution

$$
y(x)=\frac{1}{\sqrt{c-x}},
$$

whose existence interval is $[0, c)$.

However, from the proof of Theorem 3.1 we can see that if the Lipschitz constant $L$ is small enough, then $x_{0}$ can be extended to the whole interval. Thus we have the following result.

Theorem 3.2: Let $0<\alpha<1$, and $G=[a, b] \times \mathbf{X}$. Let $f: G \rightarrow \mathbf{X}$ be continuous and fulfil a Lipschitz con- 
dition with respect to the second variable with a Lipschitz constant $L$. If $L<\frac{\Gamma(\alpha+1)}{2(b-a)^{\alpha}}$, then for every

$\left(x_{0}, y_{0}\right) \in G$, there exists an $h>0$ with $x_{0}+h \in[a, b]$ and a unique mild solution $y \in C\left(\left[a, x_{0}+h\right] ; \mathbf{X}\right)$ to the IVP (1.1)-(1.2).

Next we want to study the case that $f$ satisfies the Carathedory condition. For simplicity, we limit to the case that $f$ is locally bounded. We list the hypotheses.

$\left(\mathrm{H}_{1}\right): \quad f:[a, b] \times \mathbf{X} \rightarrow \mathbf{X}$ satisfies the Carathedory condition, i.e. $f(\cdot, y):[a, b] \rightarrow \mathbf{X}$ is measurable for every $y \in \mathbf{X}$ and $f(x, \cdot): \mathbf{X} \rightarrow \mathbf{X}$ is continuous for almost every $x \in[a, b]$.

$\left(\mathrm{H}_{2}\right)$ : For every $r>0$, there is a constant $M_{r}>0$, such that $\|f(x, y)\| \leq M_{r}$ for a.e. $x \in[a, b]$ and $y \in \mathbf{X}$ with $\|y\| \leq r$.

$\left(\mathrm{H}_{3}\right)$ : There exists $\eta \in L^{p}\left([a, b] ; \mathbf{R}^{+}\right)$with $p>\frac{1}{\alpha}$ such that

$$
\beta(f(x, D)) \leq \eta(x) \beta(D)
$$

for a.e. $x \in[a, b]$ and any bounded subset $D \subset \mathbf{X}$.

Theorem 3.3: Let $0<\alpha<1$ and $G=[a, b] \times \mathbf{X}$. Assume that the hypotheses $\left(H_{1}\right)-\left(H_{2}\right)$ hold, and suppose $\left(x_{0}, y_{0}\right) \in G$ satisfying

$$
x_{0}-a<\frac{\Gamma(\alpha)(p \alpha-1)}{2\|\eta\|_{p}(p-1)} .
$$

Further assume that there exists a real number $r>0$ solving the inequality

$$
\frac{\left(x_{0}-a\right)^{\alpha}}{\Gamma(\alpha+1)} \frac{M_{r}}{r}<\frac{1}{2} .
$$

Then there exists an $h>0$ such that the IVP (1.1)-(1.2) has at least a solution $y \in C\left(\left[a, x_{0}+h\right] ; \mathbf{X}\right)$.

Proof. On account of the hypothesis (3.8), we can find constants $r_{0}>0$ large enough and $h>0$ with

$$
\left\|y_{0}\right\|+\frac{2\left(x_{0}+h-a\right)^{\alpha} M_{r_{0}}}{\Gamma(\alpha+1)}<r_{0} .
$$

Due to the hypothesis (3.6), we can take $h>0$ small enough such that

$$
x_{0}+h-a<\frac{\Gamma(\alpha)(p \alpha-1)}{2\|\eta\|_{p}(p-1)} .
$$

Define an operator $T: C\left(\left[a, x_{0}+h\right] ; \mathbf{X}\right) \rightarrow C\left(\left[a, x_{0}+h\right] ; \mathbf{X}\right)$ by

$$
T y(x)=y_{0}+\frac{1}{\Gamma(\alpha)}\left(\int_{a}^{x}(x-t)^{\alpha-1} f(t, y(t)) \mathrm{d} t-\int_{a}^{x_{0}}\left(x_{0}-t\right)^{\alpha-1} f(t, y(t)) \mathrm{d} t\right)
$$

for $y \in C\left(\left[a, x_{0}+h\right] ; \mathbf{X}\right)$ and $x \in\left[a, x_{0}+h\right]$. It then follows from the hypotheses $\left(\mathrm{H}_{1}\right)-\left(\mathrm{H}_{2}\right)$ as well as the Lebesgue dominated convergence theorem that $T$ is well-defined, i.e., Ty is continuous on $\left[a, x_{0}+h\right]$ for every $y \in C\left(\left[a, x_{0}+h\right] ; \mathbf{X}\right)$, and that $T$ is continuous. Further, let $B_{r_{0}}=\left\{y \in C\left(\left[a, x_{0}+h\right] ; \mathbf{X}\right):\|y\|_{\infty} \leq r_{0}\right\}$. Then $B_{r_{0}}$ is a bounded closed subset of $C\left(\left[a, x_{0}+h\right] ; \mathbf{X}\right)$. For every $y \in B_{r_{0}}$ and $x \in\left[a, x_{0}+h\right]$, we have

$$
\begin{aligned}
\|T y(x)\| & \leq\left\|y_{0}\right\|+\frac{1}{\Gamma(\alpha)}\left(\int_{a}^{x}(x-t)^{\alpha-1}\|f(t, y(t))\| \mathrm{d} t+\int_{a}^{x_{0}}\left(x_{0}-t\right)^{\alpha-1}\|f(t, y(t))\| \mathrm{d} t\right) \\
& \leq\left\|y_{0}\right\|+\frac{M_{r_{0}}}{\Gamma(\alpha)}\left(\int_{a}^{x}(x-t)^{\alpha-1} \mathrm{~d} t+\int_{a}^{x_{0}}\left(x_{0}-t\right)^{\alpha-1} \mathrm{~d} t\right) \leq\left\|y_{0}\right\|+\frac{2 M_{r_{0}}\left(x_{0}+h-a\right)^{\alpha}}{\Gamma(\alpha+1)} \leq r_{0}
\end{aligned}
$$

due to $\left(\mathrm{H}_{2}\right)$ and (3.8) which implie that $T B_{r_{0}} \subset B_{r_{0}}$.

Below we show that $T$ satisfies the hypotheses of Darbo-Sadovskii Theorem (Lemma 2.5). We first prove that 
$T$ maps bounded subsets in $C\left(\left[a, x_{0}+h\right], \mathbf{X}\right)$ into bounded subsets. For this purpose we show that $T B_{r}$ is bounded for every $B_{r}=\left\{y \in C\left(\left[a, x_{0}+h\right] ; \mathbf{X}\right):\|y\|_{\infty} \leq r\right\}$ with fixed $r>0$. Let $y \in B_{r}$. Then by $\left(\mathrm{H}_{2}\right)$, for every $x \in\left[a, x_{0}+h\right]$, we have

$$
\begin{aligned}
\|T y(x)\| & \leq\left\|y_{0}\right\|+\frac{1}{\Gamma(\alpha)}\left(\int_{a}^{x}(x-t)^{\alpha-1}\|f(t, y(t))\| \mathrm{d} t+\int_{a}^{x_{0}}\left(x_{0}-t\right)^{\alpha-1}\|f(t, y(t))\| \mathrm{d} t\right) \\
& \leq\left\|y_{0}\right\|+\frac{M_{r}}{\Gamma(\alpha)}\left(\int_{a}^{x}(x-t)^{\alpha-1} \mathrm{~d} t+\int_{a}^{x_{0}}\left(x_{0}-t\right)^{\alpha-1} \mathrm{~d} t\right) \leq\left\|y_{0}\right\|+\frac{2 M_{r} b^{\alpha}}{\Gamma(\alpha+1)} .
\end{aligned}
$$

It follows that $\|T y\|_{\infty} \leq\left\|y_{0}\right\|+\frac{2 M_{r} b^{\alpha}}{\Gamma(\alpha+1)}$ which is independent of $y \in B_{r}$. Hence $T B_{r}$ is bounded.

Next we prove that $T$ maps bounded subsets into equi-continuous subsets. Let $y \in B_{r}$ be arbitrary and $x_{1}, x_{2} \in\left[a, x_{0}+h\right]$ with $x_{1}<x_{2}$. Then we have

$$
\begin{aligned}
& \left\|\operatorname{Ty}\left(x_{2}\right)-T y\left(x_{1}\right)\right\| \\
& \leq \frac{1}{\Gamma(\alpha)}\left(\int_{a}^{x_{1}} \mid\left(x_{2}-t\right)^{\alpha-1}-\left(x_{1}-t\right)^{\alpha-1}\|f(t, y(t))\| \mathrm{d} t+\int_{x_{1}}^{x_{2}}\left(x_{2}-t\right)^{\alpha-1}\|f(t, y(t))\| \mathrm{d} t\right) \\
& \leq \frac{M_{r}}{\Gamma(\alpha)}\left(\int_{a}^{x_{1}}\left[\left(x_{1}-t\right)^{\alpha-1}-\left(x_{2}-t\right)^{\alpha-1}\right] \mathrm{d} t+\int_{x_{1}}^{x_{2}}\left(x_{2}-t\right)^{\alpha-1} \mathrm{~d} t\right) \\
& \leq \frac{M_{r}}{\Gamma(\alpha+1)}\left[\left(x_{2}-a\right)^{\alpha}-\left(x_{1}-a\right)^{\alpha}\right],
\end{aligned}
$$

which converges to 0 as $x_{2}-x_{1} \rightarrow 0$, and the convergence is independent of $y \in B_{r}$. Thus $T B_{r}$ is equicontinuous.

Now we verify that $T$ is a $\beta_{c}$-contraction. Take any bounded subset $W \subset T B_{r_{0}}$, then $W$ is equi-continuous. So we get from Lemma 2.4, 2.6 and 2.8 that

$$
\begin{aligned}
\beta_{c}(T W) & =\sup _{x \in\left[a, x_{0}+h\right]} \beta(T W(x)) \\
& =\sup _{x \in\left[a, x_{0}+h\right]} \frac{1}{\Gamma(\alpha)} \beta\left(\int_{a}^{x}(x-t)^{\alpha-1} f(t, W(t)) \mathrm{d} t-\int_{a}^{x_{0}}\left(x_{0}-t\right)^{\alpha-1} f(t, W(t)) \mathrm{d} t\right) \\
& \left.\leq \sup _{x \in\left[a, x_{0}+h\right]} \frac{1}{\Gamma(\alpha)}\left(\int_{a}^{x}(x-t)^{\alpha-1} \beta(f(t, W(t))) \mathrm{d} t+\int_{a}^{x_{0}}\left(x_{0}-t\right)\right)^{\alpha-1} \beta(f(t, W(t))) \mathrm{d} t\right) \\
& \leq \sup _{x \in\left[a, x_{0}+h\right]} \frac{1}{\Gamma(\alpha)}\left(\int_{a}^{x}(x-t)^{\alpha-1} \eta(t) \beta(W(t)) \mathrm{d} t+\int_{a}^{x_{0}}\left(x_{0}-t\right)^{\alpha-1} \eta(t) \beta(W(t)) \mathrm{d} t\right) \\
& \leq \sup _{x \in\left[a, x_{0}+h\right]} \frac{\beta_{c}(W)}{\Gamma(\alpha)}\left(\int_{a}^{x}(x-t)^{\alpha-1} \eta(t) \mathrm{d} t+\int_{a}^{x_{0}}\left(x_{0}-t\right)^{\alpha-1} \eta(t) \mathrm{d} t\right) .
\end{aligned}
$$

The assumption $p>\frac{1}{\alpha}$ implies that $\frac{(\alpha-1) p}{p-1}>-1$, which shows that the function $(x-\cdot)^{\alpha-1} \in L^{q}(a, x)$ with $q=\frac{p}{p-1}$ for every $x \in(a, b]$. Hence an employment of Hölder inequality yields

$$
\begin{aligned}
\beta_{c}(T W) & \leq \sup _{x \in\left[a, x_{0}+h\right]} \frac{\beta_{c}(W)\|\eta\|_{p}}{\Gamma(\alpha)}\left(\left(\int_{a}^{x}(x-t)^{\frac{(\alpha-1) p}{p-1}} \mathrm{~d} t\right)^{\frac{p-1}{p}}+\left(\int_{a}^{x_{0}}\left(x_{0}-t\right)^{\frac{(\alpha-1) p}{p-1}} \mathrm{~d} t\right)^{\frac{p-1}{p}}\right) \\
& \leq \frac{2(p-1)}{p \alpha-1}\left(x_{0}+h-a\right)^{\frac{p \alpha-1}{p-1}} \frac{\|\eta\|_{p}}{\Gamma(\alpha)} \beta_{c}(W) \\
& \leq \frac{2(p-1)}{p \alpha-1}\left(x_{0}+h-a\right) \frac{\|\eta\|_{p}}{\Gamma(\alpha)} \beta_{c}(W) .
\end{aligned}
$$


From the inequality (3.9), we deduce that $\frac{2(p-1)}{p \alpha-1}\left(x_{0}+h-a\right) \frac{\|\eta\|_{p}}{\Gamma(\alpha)}<1$, which means that $T$ is a $\beta_{c}$-contraction on $T B_{r_{0}}$.

We have now shown that $T B_{r_{0}} \subset B_{r_{0}}$ that $T$ maps bounded subsets into bounded and equi-continuous subsets, and that $T$ is a $\beta_{c}$-contraction on $T B_{r_{0}}$. By Darbo-Sadovskii Theorem (Lemma 2.5), we conclude that $T$ has at least a fixed point $y$ in $T B_{r_{0}}$, which is the solution to (1.1)-(1.2) on $\left[a, x_{0}+h\right]$, and the proof is completed.

\section{Acknowledgements}

This research was supported by the National Natural Science Foundation of China (11271316, 11571300 and 11201410) and the Natural Science Foundation of Jiangsu Province (BK2012260).

\section{References}

[1] Diethelm, K. (2010) The Analysis of Fractional Differential Equations. Lecture Notes in Mathematics, Springer-Verlag, Berlin. http://dx.doi.org/10.1007/978-3-642-14574-2

[2] Diethelm, K. and Ford, N.J. (2002) Analysis of Fractional Differential Equations. Journal of Mathematical Analysis and Applications, 265, 229-248. http://dx.doi.org/10.1006/jmaa.2000.7194

[3] Kilbas, A.A., Srivastava, H.M. and Trujillo, J.J. (2006) Theory and Applications of Fractional Differential Equations. North-Holland Mathematics Studies, Elsevier Science B.V., Amsterdam.

[4] Delbosco, D. and Rodino, L. (1996) Existence and Uniqueness for a Nonlinear Fractional Differential Equation. Journal of Mathematical Analysis and Applications, 204, 609-625. http://dx.doi.org/10.1006/jmaa.1996.0456

[5] Lakshmikantham, V. and Devi, J.V. (2008) Theory of Fractional Differential Equations in Banach Space. European Journal of Pure and Applied Mathematics, 1, 38-45.

[6] Akmerov, R.R., Kamenski, M.I., Potapov, A.S., Rodkina, A.E. and Sadovskii, B.N. (1992) Measure of Noncompactness and Condensing Operators. Birkhauser Verlag, Basel. http://dx.doi.org/10.1007/978-3-0348-5727-7

[7] Ayerbe Toledano, J.M., Dominguez Benavides, T. and Lopez Acedo, G. (1997) Measure of Noncompactness in Metric Fixed Point Theory. Birkhauser Verlag, Basel. http://dx.doi.org/10.1007/978-3-0348-8920-9

[8] Banas, J. and Goebel, K. (1980) Measure of Noncompactness in Banach Spaces. Lecture Notes in Pure and Applied Mathmatics, Dekker, New York.

[9] Banas, J. and Sadarangani, K. (2008) On Some Measures of Noncompactness in the Space of Continuous Functions. Nonlinear Analysis: Theory, Methods \& Applications, 68, 377-383. http://dx.doi.org/10.1016/j.na.2006.11.003

[10] Dong, Q., Fan, Z. and Li, G. (2008) Existence of Solutions to Nonlocal Neutral Functional Differential and Integrodifferential Equations. International Journal of Nonlinear Science, 5, 140-151.

[11] Dong, Q. and Li, G. (2015) Measure of Noncompactness and Semilinear Nonlocal Functional Differential Equations in Banach Spaces. Acta Mathematica Sinica, 31, 140-150. http://dx.doi.org/10.1007/s10114-015-3097-z

[12] Dong, Q. and Li, G. (2009) Existence of Solutions for Semilinear Differential Equations with Nonlocal Conditions in Banach Spaces. Electronic Journal of Qualitative Theory of Differential Equations, 2009, 1-13. http://dx.doi.org/10.14232/ejqtde.2009.1.47

[13] Dong, Q., Li, G. and Zhang, J. (2008) Quasilinear Nonlocal Intergrodifferential Equations in Banach Spaces. Electronic Journal of Differential Equations, 2008, 1-8.

[14] Heinz, H.P. (1983) On the Behavior of Measure of Noncompactness with Respect to Differentiation and Integration of Vector-Valued Functions. Nonlinear Analysis TMA, 7, 1351-1371. http://dx.doi.org/10.1016/0362-546X(83)90006-8

[15] Kamenskii, M., Obukhovskii, V. and Zecca, P. (2001) Condensing Multivalued Maps and Semilinear Differential Inclusions in Banach Spaces. Walter de Gruyter, Berlin. http://dx.doi.org/10.1515/9783110870893 